\title{
Safety, Tolerability, and Pharmacokinetics of the Novel Anti-influenza Agent Baloxavir Marboxil in Healthy Adults: Phase I Study Findings
}

\author{
Hiroki Koshimichi ${ }^{1} \cdot$ Toru Ishibashi $^{1} \cdot$ Nao Kawaguchi $^{1} \cdot$ Chisako Sato $^{2} \cdot$ Akira Kawasaki $^{3} \cdot$ Toshihiro Wajima $^{1}$
}

Published online: 4 October 2018

(c) The Author(s) 2018

\begin{abstract}
Background and Objective Baloxavir marboxil, a prodrug that is metabolized to baloxavir acid, suppresses viral replication by inhibiting cap-dependent endonuclease. This first-in-human phase I study evaluated the safety, tolerability, and pharmacokinetics of baloxavir marboxil/baloxavir acid in healthy Japanese volunteers (Study 1), while food effects were evaluated in a separate phase I, crossover study in healthy Japanese volunteers (Study 2).

Methods Study 1 participants were randomized to single-dose oral baloxavir marboxil $(6,20,40,60$, or $80 \mathrm{mg} ; n=6$ per dose) or placebo $(n=10)$, while Study 2 participants $(n=15)$ received single-dose oral baloxavir marboxil $20 \mathrm{mg}$ in fasted, fed, and before-meal states.

Results Baloxavir marboxil was well tolerated; there were few treatment-emergent adverse events and no serious adverse events/deaths. The mean plasma baloxavir acid concentration $24 \mathrm{~h}$ after single-dose $\left(C_{24}\right)$ oral baloxavir marboxil $6 \mathrm{mg}$ was $6.92 \mathrm{ng} / \mathrm{mL}$, exceeding the target $C_{24}(6.85 \mathrm{ng} / \mathrm{mL})$ estimated in nonclinical studies. In Study 1, baloxavir acid exposure demonstrated dose-proportional increases in the fasted state, with maximum plasma concentration generally attained within $3.5 \mathrm{~h}$. Terminal elimination half-life ranged from 49 to $91 \mathrm{~h}$. In Study 2, exposure was decreased and apparent clearance increased in the fed and before-meal states versus the fasted state; however, exposure exceeded the target $C_{24}$ in all states. Conclusion Single-dose oral baloxavir marboxil was well tolerated, had a favorable safety profile, and had favorable pharmacokinetic characteristics, including a long half-life, supporting single oral dosing. The baloxavir acid area under the plasma concentration-time curve decreased with food intake by approximately $40 \%$.
\end{abstract}

\section{Key Points}

Baloxavir marboxil was well tolerated and had a generally favorable safety profile up to an $80 \mathrm{mg}$ single oral dose.

The pharmacokinetic profile showing a long half-life supports a single oral dose.

The exposure of baloxavir acid decreased under the fed state.

Toru Ishibashi

toru.ishibashi@shionogi.co.jp

1 Project Management Department, Shionogi \& Co., Ltd, Osaka, Japan

2 Clinical Research Department, Shionogi \& Co., Ltd, Osaka, Japan

3 Medical Science Department, Shionogi \& Co., Ltd, Osaka, Japan

\section{Introduction}

Influenza virus infections occur on a seasonal epidemic basis and, less commonly, in the form of global pandemics. Although symptoms are typically mild, the risks of morbidity and mortality associated with influenza are increased in high-risk groups, such as the young, the elderly, pregnant women, and individuals who are immunocompromised or have other medical comorbidities [1]. Indeed, recent estimates suggest that there are up to approximately 650,000 influenza-associated respiratory deaths annually worldwide [2]; hence, having effective influenza treatments available is critical.

Adamantanes and neuraminidase inhibitors (NAIs) are approved antivirals for the treatment of influenza; however, both have limitations. Notably, adamantanes are not recommended for treating influenza as current strains have shown significant resistance [3]. NAIs are widely used and recommended for the treatment of influenza in high-risk patients [3]; however, these antivirals are limited in that they are 
most effective when administered within $48 \mathrm{~h}$ of symptom onset [4] and may be associated with insufficient decreases in virus titers and prolonged virus shedding [5-7]. The latter may result in decreased virologic and clinical benefit. Furthermore, strain mutations in seasonal or avian viruses have led to the development of NAI resistance $[8,9]$. Thus, there is a clear need for alternative antiviral drugs, not only for the treatment of seasonal influenza but also to have available as a treatment option for future pandemics.

Baloxavir marboxil (previously known as S-033188) is a first-in-class antiviral prodrug that is metabolized to a small molecule active form (baloxavir acid [previously known as S-033447]), which in turn suppresses viral replication. Specifically, baloxavir acid inhibits cap-dependent endonuclease, and thereby the 'cap-snatching' step in the initiation of viral genome transcription. In contrast, NAIs act by inhibiting the release of new viral particles. Nonclinical data from in vitro and in vivo studies showed that baloxavir marboxil and baloxavir acid have rapid and profound antiviral activity against both type A and B influenza virus, with no crossresistance to NAIs (unpublished laboratory data).

In nonclinical studies, baloxavir marboxil is a prodrug and is converted to an active form, baloxavir acid, by hydrolysis. After single oral administration in rats and monkeys, plasma concentrations of baloxavir marboxil were below the lower limit of quantification (LLOQ) at all sampling points, and area under the plasma concentration-time curve of baloxavir acid increased dose proportionally. The major excretion route of radioactivity was via fecal excretion, whereas urinary excretion was low in rats and monkeys (unpublished laboratory data).

The aim of this first-in-human phase I study was to evaluate the safety, tolerability, and pharmacokinetics of baloxavir marboxil/baloxavir acid in healthy Japanese volunteers. We also report findings from a subsequent phase I study that evaluated the effect of food on the safety, tolerability, and pharmacokinetics of baloxavir marboxil/baloxavir acid in healthy Japanese volunteers.

\section{Methods}

\subsection{Participants}

Both studies included healthy male Japanese volunteers aged $\geq 20$ to $\leq 59$ years with a body weight $\geq 50$ to $\leq 80 \mathrm{~kg}$ and a body mass index $\geq 18.5$ to $<25.0 \mathrm{~kg} / \mathrm{m}^{2}$. Key exclusion criteria were consumption of any drug (prescription or over-the-counter drugs, dietary supplements, or vitamins) within 14 days prior to admission or 3 days prior to screening; use of alcohol-containing, caffeine-containing, grapefruit-containing, or St. John's wort-containing products within $72 \mathrm{~h}$ prior to admission; and use of tobacco- or nicotine-containing products within 24 weeks prior to screening.

All volunteers provided written informed consent prior to screening.

\subsection{Study Design}

The Institutional Review Board reviewed and approved both study protocols and sample informed consent form/written information for participants prior to screening/enrollment.

Both studies were conducted in compliance with International Conference on Harmonisation Guidelines and Good Clinical Practice and the ethical principles outlined in the Declaration of Helsinki.

\subsubsection{Study 1}

Study 1 was a randomized, placebo-controlled, double-blind, single-center study in Japan. Participants were randomized (using computer-generated random numbers) to one of five baloxavir marboxil single oral dose cohorts $(6,20,40,60$, or $80 \mathrm{mg}$ ) or placebo in the fasted state $(\geq 10 \mathrm{~h})$. Baloxavir marboxil was supplied by Shionogi \& Co., Ltd, and prepared as a $20 \mathrm{~mL}$ oral suspension. Each baloxavir marboxil dose level could be administered only after satisfactory review of safety, tolerability, and pharmacokinetic data from the preceding dose group.

The study included the following: screening (days -28 to -2 ), admission to the study center (day -1 ), administration of the study drug (day 1), discharge from the study center (day 4), post-treatment observation (day 6), follow-up 1 (day 8 ), and follow-up 2 (day 15; for participants in the 60 and $80 \mathrm{mg}$ cohorts only).

Blood for pharmacokinetic analysis was sampled predose and $0.5,1,1.5,2,2.5,3,3.5,4,5,6,8$, and $12 \mathrm{~h}$ after drug administration on day 1 . Thereafter, sampling was every $12 \mathrm{~h}$ through $72 \mathrm{~h}$, and, for participants who received the 40 , 60 , and $80 \mathrm{mg}$ doses, at $336 \mathrm{~h}$. At each point, a $4 \mathrm{~mL}$ blood sample was collected in a heparinized tube and centrifuged for 10 min under $4{ }^{\circ} \mathrm{C}, 2000 \times g$ soon after the sample collection to separate the plasma. Urine for pharmacokinetic analysis was sampled predose ( -12 to $0 \mathrm{~h}$ ) and $0-12,12-24$, 24-48, and 48-72 h after drug administration on day 1.

\subsubsection{Study 2}

Study 2 was a randomized, three-dosing sequence, threeperiod, crossover food effects study in Japan that was carried out at the same site as Study 1. Randomization was via computer-generated random numbers. During each period, participants received a single, 20-mg oral dose of baloxavir marboxil in tablet form in the fasted $(\geq 10 \mathrm{~h}$ ), fed (30 min after eating), or before-meal state (after a fast of $\geq 10 \mathrm{~h}$ and 
$1 \mathrm{~h}$ before the initiation of a meal). Baloxavir marboxil was supplied by Shionogi \& Co., Ltd, and prepared as a $20-\mathrm{mg}$ tablet, which was the highest strength. There was a washout period of at least 21 days between periods. The predose meal for administration in the fed state, and the postdose meal for administration in the before-meal state, had approximately $400-500 \mathrm{kcal}$, of which approximately $150 \mathrm{kcal}$ was derived from fat.

After initial screening (days -28 to -2 ), each study period included the following: admission to the study center (day -1 ), administration of the study drug (day 1), discharge from the study center (day 4), post-treatment observation (day 6), follow-up 1 (day 8), follow-up 2 (day 15), follow-up 3 (day 22; for period 3 only).

Blood for pharmacokinetic analysis was sampled predose and $1,2,3,4,5,6,8$, and $12 \mathrm{~h}$ after drug administration on day 1 . Thereafter, sampling was every $12 \mathrm{~h}$ through $48 \mathrm{~h}$, and then at 72, 120, 168, 336, and $504 \mathrm{~h}$. At each point, a $3 \mathrm{~mL}$ blood sample was collected in a heparinized tube and centrifuged for 10 min under $4{ }^{\circ} \mathrm{C}, 2000 \times g$ soon after the sample collection to separate the plasma.

\subsection{Bioanalytical Assay}

The bioanalytical methods for the determination of plasma concentrations of baloxavir marboxil and baloxavir acid were validated, where the LLOQ was set at $0.100 \mathrm{ng} / \mathrm{mL}$. Dichlorvos, as an inhibitor of esterases that metabolize baloxavir marboxil to baloxavir acid, was added to the plasma samples. Plasma samples were analyzed after deproteinization using the liquid chromatography-tandem mass spectrometry (LCMS/MS) method. The assay was linear from 0.100 to $300 \mathrm{ng} /$ $\mathrm{mL}$. The precision levels of the assay were $2.8-7.4 \%$ and 2.4-6.1\% for baloxavir marboxil and baloxavir acid, respectively, and the accuracy levels of the assay were $2.0-6.0 \%$ and -1.5 to $6.8 \%$ for baloxavir marboxil and baloxavir acid, respectively.

The bioanalytical methods for the determination of urine concentrations of baloxavir marboxil and baloxavir acid were validated, where the LLOQ was set at $0.100 \mathrm{ng} /$ $\mathrm{mL}$. Urine samples were analyzed after deproteinization using the LC-MS/MS method. The assay was linear from 0.100 to $300 \mathrm{ng} / \mathrm{mL}$. The precision levels of the assay were $1.6-11.6 \%$ and $1.1-6.5 \%$ for baloxavir marboxil and baloxavir acid, respectively, and the accuracy levels of the assay were -0.6 to $2.7 \%$ and -4.7 to $2.8 \%$ for baloxavir marboxil and baloxavir acid, respectively.

\subsection{Safety Assessments}

Safety assessments performed throughout both studies included the recording of treatment-emergent adverse events (TEAEs), physical examinations, laboratory tests, vital signs, and electrocardiograms. TEAEs were classified per the Medical Dictionary for Regulatory Activities (version 17.1). A drug-related TEAE was defined as a TEAE where it can be reasonably explained that the study drug caused the TEAE. For example, a TEAE will be deemed related to the study drug if the occurrence of the TEAE cannot be explained by other causative factors, can be explained by the pharmacological effect of the study drug based on a previously reported similar event, or if an increase/decrease of the dose affects the occurrence or seriousness of the TEAE. A TEAE was judged not drug related if it could not be reasonably explained that the study drug caused the TEAE.

\subsection{Pharmacokinetic Assessments}

Baloxavir acid pharmacokinetic parameters assessed in both studies included maximum plasma concentration $\left(C_{\max }\right)$, area under the plasma concentration-time curve from time zero to infinity $\left(\mathrm{AUC}_{\infty}\right)$, terminal elimination half-life $\left(t_{1 / 2, z}\right)$, apparent total clearance $(\mathrm{CL} / \mathrm{F})$, and plasma concentration $24 \mathrm{~h}$ after dosing $\left(C_{24}\right)$. Pharmacokinetic parameters assessed in Study 1 also included AUC from time zero to $72 \mathrm{~h}\left(\mathrm{AUC}_{72}\right)$ and the urinary excretion ratio relative to dose from time zero to $72 \mathrm{~h}$.

Pharmacokinetic analyses were performed using Phoenix WinNonlin version 6.2.1 (Certara USA Inc., Princeton, NJ, USA). The pharmacokinetic parameters were calculated using the linear-up/log-down trapezoidal method.

\subsection{Statistical Analysis}

There were no formal sample size calculations for either study. Study 1 included 40 participants (six active and two placebo per cohort), which is a typical number of participants for a phase I study of this nature. Study 2 included 15 participants, which meets the US FDA guidance that a minimum of 12 participants should complete food-effects studies [10].

Analysis populations for both studies were as follows: safety $=$ all participants who received at least one dose of the study drug; pharmacokinetic concentration $=$ all participants who received at least one dose of the study drug and underwent plasma pharmacokinetic sampling and had at least one evaluable pharmacokinetic assay result; pharmacokinetic parameters $=$ all participants who had at least one pharmacokinetic parameter estimated.

Continuous variables are summarized using means and standard deviation, whereas categorical variables are summarized using frequency counts and percentages. Pharmacokinetic parameters are summarized using the geometric mean and coefficient of variation for geometric mean. 


\section{Results}

\subsection{Demographic and Baseline Characteristics}

\subsubsection{Study 1}

A total of 40 Japanese participants were enrolled and were randomized to the single oral dose cohorts; all completed the study. Demographic and other baseline characteristics were well balanced between dose groups (Table 1).

\subsubsection{Study 2}

A total of 15 Japanese participants were enrolled and were randomized to the three dosing sequence cohorts (five to each cohort); 12 completed the study. Three participants received baloxavir marboxil in the fed state but were withdrawn due to TEAEs before receiving baloxavir marboxil in the fed and before-meal states. One of these participants experienced increases in aspartate aminotransferase (46 IU/L) and alanine aminotransferase (ALT, $48 \mathrm{IU} / \mathrm{L})$ that

Table 1 Demographic and baseline characteristics

\begin{tabular}{|c|c|c|c|c|c|c|c|c|c|}
\hline \multirow{3}{*}{$\begin{array}{l}\text { Character- } \\
\text { istic }\end{array}$} & \multicolumn{6}{|l|}{ Study 1} & \multicolumn{3}{|l|}{ Study 2} \\
\hline & \multicolumn{5}{|c|}{ Baloxavir marboxil $^{\mathrm{a}}$} & \multirow[b]{2}{*}{$\begin{array}{l}\text { Placebo } \\
{[N=10]}\end{array}$} & \multicolumn{3}{|c|}{ Baloxavir marboxil $20 \mathrm{mg}^{\mathrm{b}}$} \\
\hline & $6 \mathrm{mg}[N=6]$ & $\begin{array}{l}20 \mathrm{mg} \\
{[N=6]}\end{array}$ & $\begin{array}{l}40 \mathrm{mg} \\
{[N=6]}\end{array}$ & $\begin{array}{l}60 \mathrm{mg} \\
{[N=6]}\end{array}$ & $\begin{array}{l}80 \mathrm{mg} \\
{[N=6]}\end{array}$ & & $\begin{array}{l}\text { Fasted } \\
{[N=15]}\end{array}$ & Fed $[N=12]$ & $\begin{array}{l}\text { Before meal } \\
{[N=12]}\end{array}$ \\
\hline Age (years) & $23.0(3.3)$ & $25.2(4.7)$ & $28.2(4.6)$ & $26.3(4.2)$ & $29.0(5.6)$ & $30.0(7.1)$ & $28.7(6.7)$ & $29.4(6.9)$ & $29.4(6.9)$ \\
\hline Height (cm) & $174.4(6.0)$ & $173.1(2.8)$ & $168.5(3.5)$ & $172.1(2.2)$ & $171.2(5.2)$ & $174.5(3.7)$ & $170.8(5.5)$ & $172.1(4.3)$ & $172.1(4.3)$ \\
\hline Weight (kg) & $67.8(8.0)$ & 65.7 (6.3) & $59.1(4.6)$ & $63.0(6.0)$ & $63.3(7.4)$ & $67.6(5.5)$ & $62.7(6.5)$ & $64.1(6.2)$ & $64.1(6.2)$ \\
\hline BMI $\left(\mathrm{kg} / \mathrm{m}^{2}\right)$ & $22.2(1.3)$ & $21.9(2.0)$ & $20.8(1.4)$ & $21.2(1.6)$ & $21.6(2.4)$ & $22.2(1.4)$ & $21.4(1.3)$ & $21.6(1.3)$ & $21.6(1.3)$ \\
\hline
\end{tabular}

Data are expressed as mean (SD)

$B M I$ body mass index, $S D$ standard deviation

${ }^{a}$ Administered as an oral suspension

${ }^{\mathrm{b}}$ Administered as a single tablet

Table 2 Summary of adverse events after single oral doses of baloxavir marboxil in the fasted state (Study 1) or fasted, fed, and before-meal states (Study 2)

\begin{tabular}{|c|c|c|c|c|c|c|c|c|c|}
\hline \multirow[t]{3}{*}{ Adverse event } & \multicolumn{6}{|l|}{ Study 1} & \multicolumn{3}{|l|}{ Study 2} \\
\hline & \multicolumn{5}{|c|}{ Baloxavir marboxil $^{\mathrm{a}}$} & \multirow[b]{2}{*}{$\begin{array}{l}\text { Placebo } \\
{[N=10]}\end{array}$} & \multicolumn{3}{|c|}{ Baloxavir marboxil $20 \mathrm{mg}^{\mathrm{b}}$} \\
\hline & $6 \mathrm{mg}[N=6]$ & $\begin{array}{l}20 \mathrm{mg} \\
{[N=6]}\end{array}$ & $\begin{array}{l}40 \mathrm{mg} \\
{[N=6]}\end{array}$ & $\begin{array}{l}60 \mathrm{mg} \\
{[N=6]}\end{array}$ & $\begin{array}{l}80 \mathrm{mg} \\
{[N=6]}\end{array}$ & & Fasted $[N=15]$ & Fed $[N=12]$ & $\begin{array}{l}\text { Before } \\
\text { meal } \\
{[N=12]}\end{array}$ \\
\hline TEAEs & $1(16.7)$ & 0 & 0 & 0 & 0 & 0 & $7(46.7)$ & $3(25.0)$ & $1(8.3)$ \\
\hline \multicolumn{10}{|l|}{ Most common TEAEs ${ }^{c}$} \\
\hline ALT increased & 0 & 0 & 0 & 0 & 0 & 0 & $1(6.7)$ & $2(16.7)$ & 0 \\
\hline Eosinophil count increased & 0 & 0 & 0 & 0 & 0 & 0 & $1(6.7)$ & $1(8.3)$ & 0 \\
\hline Headache & $1(16.7)$ & 0 & 0 & 0 & 0 & 0 & $1(6.7)$ & 0 & 0 \\
\hline White blood cell count increased & 0 & 0 & 0 & 0 & 0 & 0 & $1(6.7)$ & 0 & $1(8.3)$ \\
\hline SAEs & 0 & 0 & 0 & 0 & 0 & 0 & 0 & 0 & 0 \\
\hline Deaths & 0 & 0 & 0 & 0 & 0 & 0 & 0 & 0 & 0 \\
\hline $\begin{array}{l}\text { TEAEs leading to withdrawal of study } \\
\text { drug }\end{array}$ & 0 & 0 & 0 & 0 & 0 & 0 & $3(20.0)$ & 0 & 0 \\
\hline Treatment-related TEAEs & $1(6.7)$ & 0 & 0 & 0 & 0 & 0 & $3(20.0)$ & $2(16.7)$ & 0 \\
\hline
\end{tabular}

Data are expressed as number of participants $(\%)$

ALT alanine aminotransferase, SAEs serious adverse events, TEAEs treatment-emergent adverse events

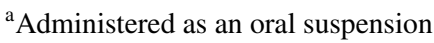

${ }^{\mathrm{b}}$ Administered as a single tablet

${ }^{\mathrm{c}}$ Experienced by one participant in Study 1 or two or more participants overall in Study 2 

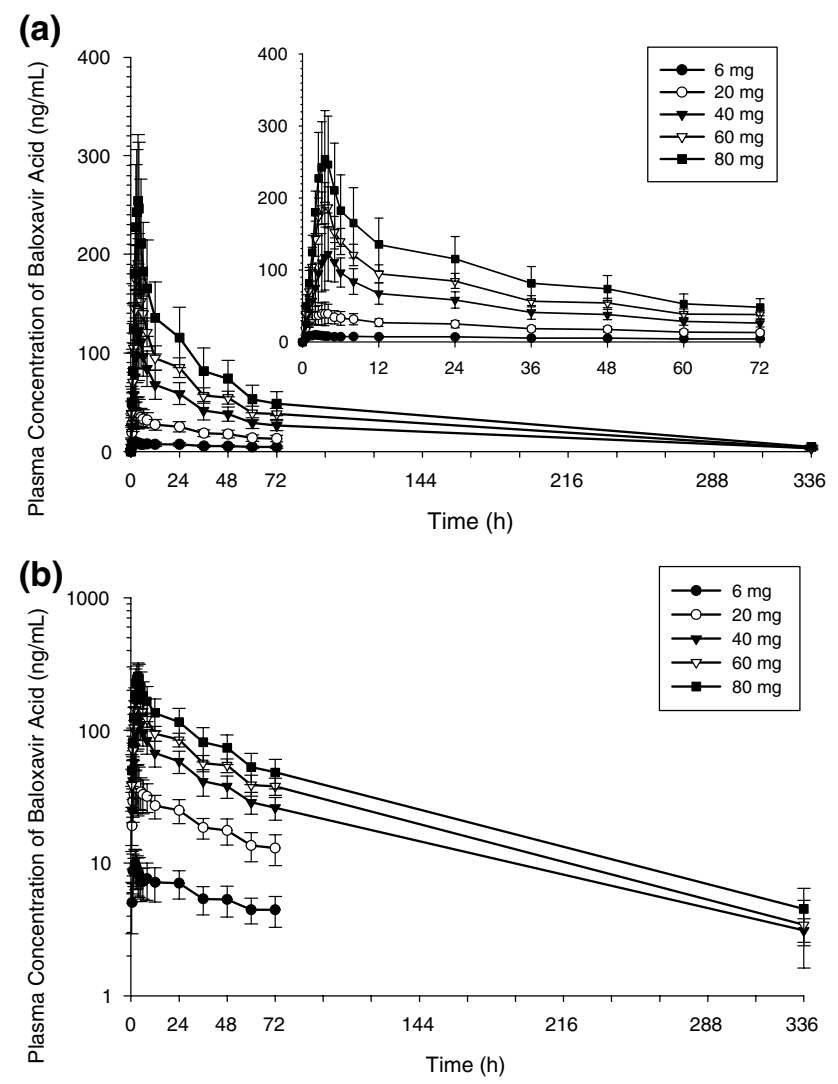

Fig. 1 Mean $( \pm S D)$ plasma baloxavir acid concentration-time profiles after single oral suspension doses of baloxavir marboxil in the fasted state (Study 1). a Linear scale (superimposes the same plasma concentration-time profile with the $x$-axis until $72 \mathrm{~h}$ ); b semi-log scale. $S D$ standard deviation

were considered to be associated with infectious mononucleosis and not related to the study drug. The remaining two participants reported TEAEs of moderate tonsillitis and mild rash, respectively, that were considered not related to the study drug.

Demographic and other baseline characteristics were well balanced between the different food dosing groups (Table 1).

\subsection{Safety and Tolerability}

Baloxavir marboxil was generally well tolerated in both studies (Table 2). Relatively few TEAEs were reported overall, and no serious adverse events (SAEs) or deaths. Only one TEAE was reported in Study 1. This TEAE, headache, was reported by a participant in the 6-mg dose group, was mild in severity, resolved without any treatment, and was considered by the investigator to be related to the study drug. TEAEs reported by two or more participants in Study 2 included ALT increased $(n=3)$, eosinophil count increased $(n=2)$, and white blood cell count increased $(n=2)$. Overall, eight treatment-related TEAEs were reported by five participants in Study 2, including ALT increased $(n=3)$, headache,

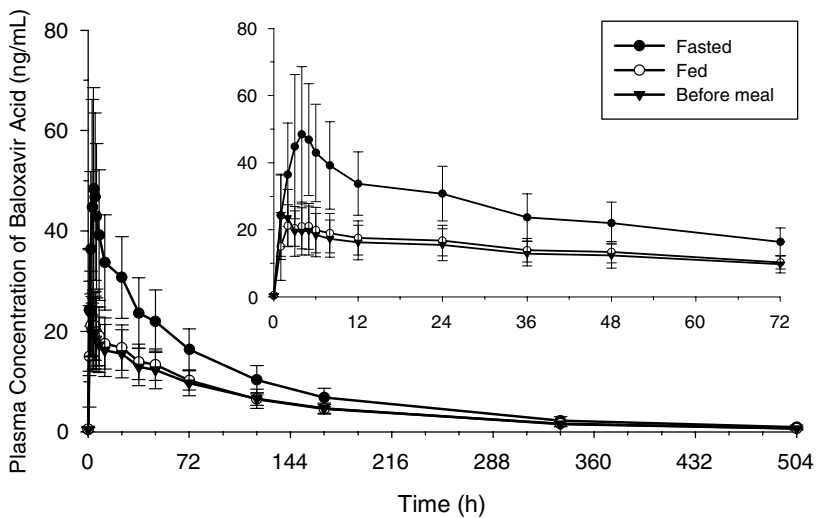

Fig. 2 Mean $( \pm S D)$ plasma baloxavir acid concentration-time profiles after single oral tablet doses of baloxavir marboxil in the fasted, fed, and before-meal states (Study 2). $S D$ standard deviation

stomatitis, and aspartate aminotransferase increased, blood lactate dehydrogenase (LDH) increased, and eosinophil count increased (all $n=1$ ). All of these events were mild in severity and, except for the event of blood LDH increased, resolved without any treatment. The event of LDH increased did not resolve during the study period.

There were no obvious trends in laboratory values, vital signs, or electrocardiogram parameters (data not shown).

\subsection{Pharmacokinetics}

\subsubsection{Plasma Concentration-Time Profiles}

In Study 1, plasma baloxavir marboxil concentrations were very low (maximum $0.363 \mathrm{ng} / \mathrm{mL}$, quantification limit $<0.100 \mathrm{ng} / \mathrm{mL}$ ) and baloxavir marboxil was rapidly eliminated from plasma in all participants (data not shown). Plasma baloxavir acid concentrations increased with increasing baloxavir marboxil dose (Fig. 1). The mean $C_{24}$ of baloxavir marboxil $6 \mathrm{mg}(6.92 \mathrm{ng} / \mathrm{mL})$ exceeded the mean target $C_{24}$ $(6.85 \mathrm{ng} / \mathrm{mL})$ estimated from nonclinical study findings [11].

In Study 2, plasma baloxavir marboxil concentrations were below the limit of quantification in all participants. Plasma baloxavir acid concentrations tended to be lower in the fed or before-meal states than in the fasted state (Fig. 2).

\subsubsection{Pharmacokinetic Parameters}

In Study 1, baloxavir acid exposure measures $\left(C_{\max }\right.$, AUC ${ }_{72}$, and $\mathrm{AUC}_{\infty}$ ) demonstrated essentially dose-proportional increases in the fasted state (Table 3, Fig. 3), with $C_{\max }$ generally attained within $3.5 \mathrm{~h}$ after dosing. The $t_{1 / 2, z}$ ranged from 49 to $91 \mathrm{~h}$, while renal excretion was minimal.

In Study 2, most baloxavir acid pharmacokinetic parameters differed by food state (Table 3). Exposure was decreased 
Table 3 Summary of pharmacokinetic parameters of plasma baloxavir acid after single oral doses of baloxavir marboxil in the fasted state (Study 1) or fasted, fed, and before-meal states (Study 2)

\begin{tabular}{|c|c|c|c|c|c|c|c|c|}
\hline \multirow[t]{3}{*}{ Parameter } & \multicolumn{5}{|l|}{ Study 1} & \multicolumn{3}{|l|}{ Study 2} \\
\hline & \multicolumn{5}{|c|}{ Baloxavir marboxil $^{\mathrm{a}}$} & \multicolumn{3}{|c|}{ Baloxavir marboxil $20 \mathrm{mg}^{\mathrm{b}}$} \\
\hline & $6 \mathrm{mg}[N=6]$ & $20 \mathrm{mg}[N=6]$ & $40 \mathrm{mg}[N=6]$ & $60 \mathrm{mg}[N=6]$ & $80 \mathrm{mg}[N=6]$ & Fasted $[N=15]$ & Fed $[N=12]$ & $\begin{array}{l}\text { Before meal } \\
{[N=12]}\end{array}$ \\
\hline$C_{\max }(\mathrm{ng} / \mathrm{mL})$ & $11.0(22.3)$ & $40.2(32.5)$ & $123(31.0)$ & $193(15.7)$ & 253 (23.9) & $49.1(34.9)$ & $25.7(21.6)$ & $25.2(37.2)$ \\
\hline $\begin{array}{l}T_{\max }(\mathrm{h}) \\
\quad[\text { median } \\
\quad(\min , \max )]\end{array}$ & $2.0(1.0,2.5)$ & $3.5(1.5,4.0)$ & $3.5(3.5,5.0)$ & $3.25(2.5,4.0)$ & $3.5(2.5,4.0)$ & $4.0(1.0,5.0)$ & $3.0(1.0,5.0)$ & $1.5(1.0,12.0)$ \\
\hline $\begin{array}{l}\operatorname{AUC}_{72}(\mathrm{ng} \cdot \mathrm{h} / \\
\mathrm{mL})\end{array}$ & $417.4(22.1)$ & $1484(21.5)$ & $3475(22.5)$ & $5073(11.8)$ & $6795(25.5)$ & - & - & - \\
\hline $\begin{array}{l}\mathrm{AUC}_{\text {last }}(\mathrm{ng} \cdot \mathrm{h} / \\
\mathrm{mL})\end{array}$ & $417.4(22.1)$ & $1484(21.5)$ & $6285(20.9)$ & $8767(15.7)$ & $11490(27.0)$ & $3697(26.1)$ & $2314(21.8)$ & $2206(26.8)$ \\
\hline $\begin{array}{l}\mathrm{AUC}_{\infty}(\mathrm{ng} \cdot \mathrm{h} / \\
\mathrm{mL})\end{array}$ & $1018(35.7)$ & $2419(24.8)$ & $6669(20.8)$ & $9141(17.5)$ & $11970(27.8)$ & 3867 (25.6) & $2429(22.4)$ & $2306(27.0)$ \\
\hline$t_{1 / 2, z}(\mathrm{~h})$ & $90.9(55.7)$ & $48.9(30.1)$ & $85.9(8.2)$ & $75.2(15.3)$ & $75.9(11.1)$ & $104(21.0)$ & $114(19.3)$ & $110(14.5)$ \\
\hline $\mathrm{CL} / F(\mathrm{~L} / \mathrm{h})$ & $4.99(35.7)$ & $6.99(24.8)$ & $5.07(20.8)$ & $5.55(17.5)$ & $5.65(27.8)$ & $4.38(25.6)$ & $6.97(22.4)$ & $7.34(27.0)$ \\
\hline$C_{24}(\mathrm{ng} / \mathrm{mL})$ & $6.92(22.1)$ & $24.4(22.5)$ & $57.6(20.1)$ & $84.4(12.7)$ & $112(27.2)$ & $29.8(25.7)$ & $16.2(28.4)$ & $14.9(32.5)$ \\
\hline $\mathrm{Feu}_{72}(\%)$ & $1.7(14.8)$ & $1.9(22.8)$ & $2.1(23.7)$ & $2.1(9.3)$ & $2.3(25.7)$ & - & - & - \\
\hline
\end{tabular}

Data are expressed as geometric mean (CV\% geometric mean) unless otherwise indicated

$A U C_{72}$ area under the concentration-time curve from time zero to $72 \mathrm{~h}, A U C_{\infty}$ area under the concentration-time curve from time zero to infinity, $A U C_{\text {last }}$ area under the concentration-time curve from time zero to the last quantifiable concentration, $C_{24}$ plasma concentration $24 \mathrm{~h}$ after dosing, $C L / F$ apparent total clearance, $C_{\max }$ maximum plasma concentration, $\mathrm{CV}$ coefficient of variation, $\mathrm{Feu}_{72}$ urinary excretion ratio relative to dose from time zero to $72 \mathrm{~h}, t_{1 / 2, z}$ terminal elimination half-life, $T_{\max }$ time to maximum plasma concentration, $\min$ minimum, max maximum

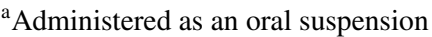

${ }^{\mathrm{b}}$ Administered as a single tablet

and $\mathrm{CL} / \mathrm{F}$ increased in the fed and before-meal states compared with the fasted state. In the fed state, $C_{\max }, \mathrm{AUC}_{\text {last }}$, and $\mathrm{AUC}_{\infty}$ were decreased by $47 \%, 37 \%$, and $37 \%$, respectively, compared with the fasted state. In the before-meal state, $C_{\text {max }}$, AUC extrapolated from time zero to the time of the last quantifiable concentration after dosing $\left(\mathrm{AUC}_{\text {last }}\right)$, and $\mathrm{AUC}_{\infty}$ were decreased by $48 \%, 40 \%$, and $39 \%$, respectively, compared with the fasted state.

\section{Discussion}

This is the first-in-human study of baloxavir marboxil, an influenza treatment with a novel mechanism of action (capdependent endonuclease inhibitor). Of note, single-dose oral baloxavir marboxil was well tolerated and was associated with encouraging safety findings in healthy Japanese male participants. Furthermore, after single oral dosing, the prodrug baloxavir marboxil was rapidly metabolized to baloxavir acid. The pharmacokinetics of baloxavir acid were linear and baloxavir acid had a long elimination half-life. In addition, Study 2 (healthy Japanese volunteers) demonstrated food effects on the pharmacokinetics of baloxavir acid. Taken together, our findings support continued clinical evaluation of baloxavir marboxil as a treatment for influenza.

We found that Japanese participants who received single oral doses of baloxavir marboxil (6-80 mg administered as an oral suspension, or $20 \mathrm{mg}$ administered in tablet form) experienced very few TEAEs in general and no SAEs or deaths. In Study 2, although eight treatment-related TEAEs were reported, all except one were mild in severity and resolved without any treatment. In addition, any subjects with abnormal laboratory findings were asymptomatic. No obvious trends were noted among the TEAEs and laboratory data. Safety and tolerability profiles were similar between the different dose cohorts. Our findings indicate that baloxavir marboxil has generally favorable safety and tolerability.

The expected therapeutic benefits of baloxavir marboxil over currently approved treatments include faster resolution of influenza symptoms and a faster cessation of infectious virus shedding due to rapid reduction in virus titer. In a nonclinical study, the target viral titer level $24 \mathrm{~h}$ after oral administration of baloxavir marboxil in mice was set to be one-tenth of that associated with oral administration of a clinically equivalent dose of oseltamivir. This target was achieved with baloxavir marboxil $15 \mathrm{mg} / \mathrm{kg}$ twice-daily dosing for 1 day. Subsequent analyses in mice revealed that 
Fig. 3 Mean $( \pm S D)$ baloxavir acid a $C_{\max }, \mathbf{b} \mathrm{AUC}_{72}$, and $\mathbf{c}$ $\mathrm{AUC}_{\infty}$ by baloxavir marboxil dose in the fasted stated (Study 1). $A U C_{72}$ area under the concentration-time curve from time zero to $72 \mathrm{~h}, A U C_{\infty}$ area under the concentration-time curve from time zero to infinity, $A U C$ extr the extrapolated percentage of $\mathrm{AUC}_{\infty}, C_{\max }$ maximum plasma concentration
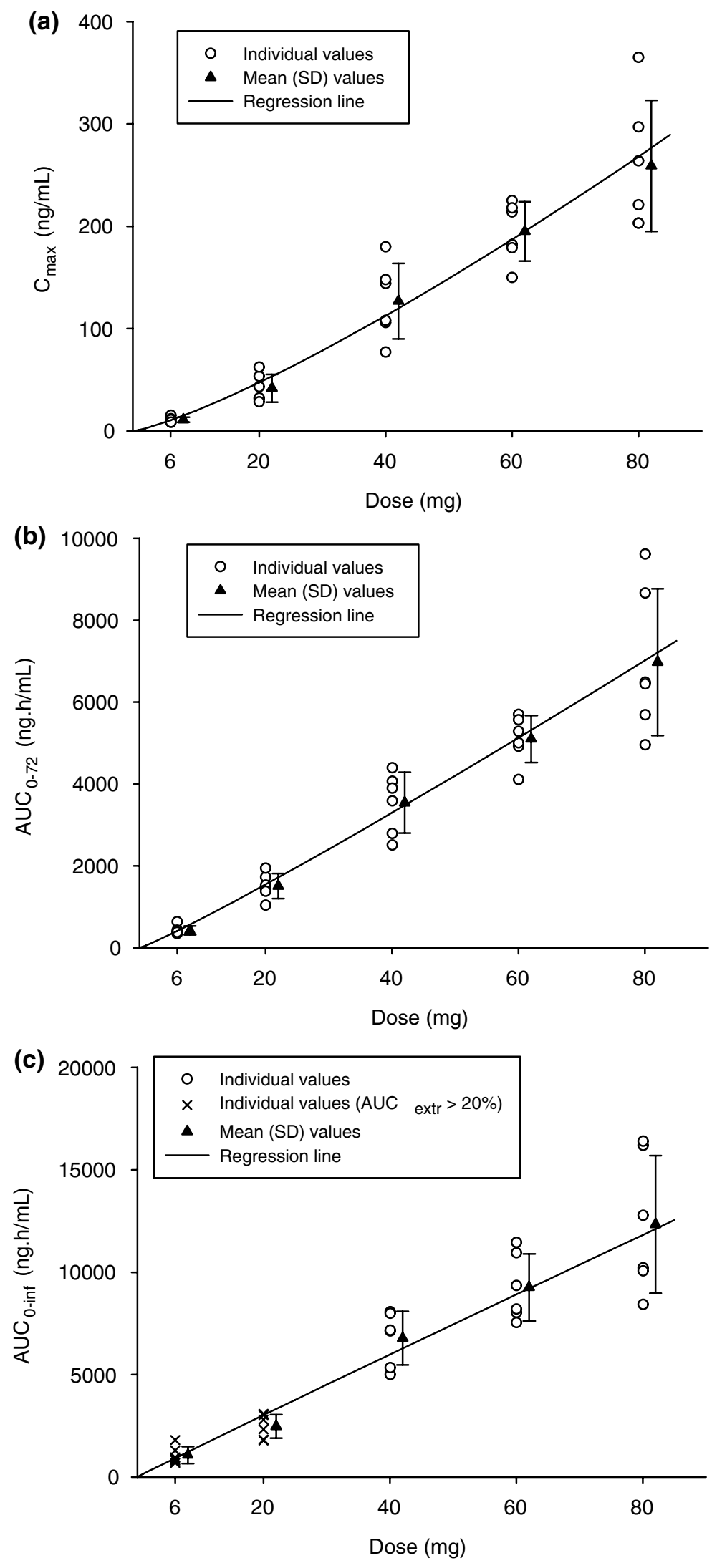
the plasma concentration of baloxavir acid at the end of the dosing interval after the first dose $\left(C_{\tau}\right)$ was the pharmacokinetic parameter that best correlated with lung virus titer the day after dosing. The $C_{\tau}$ with baloxavir marboxil $15 \mathrm{mg} / \mathrm{kg}$ twice-daily dosing was $6.85 \mathrm{ng} / \mathrm{mL}$ [11]; hence, a baloxavir acid $C_{24} \geq 6.85 \mathrm{ng} / \mathrm{mL}$ was estimated to provide superior inhibition of virus replication compared with that provided by oseltamivir. Furthermore, the 50\% effective concentration of baloxavir acid ranged from 0.63 to $0.77 \mathrm{ng} / \mathrm{mL}$ $(1.3-1.6 \mathrm{nmol} / \mathrm{L})$ for $\mathrm{A} / \mathrm{H} 1 \mathrm{~N} 1 \mathrm{pdm}$, from 0.36 to $0.68 \mathrm{ng} / \mathrm{mL}$ $(0.74-1.4 \mathrm{nmol} / \mathrm{L})$ for $\mathrm{A} / \mathrm{H} 3 \mathrm{~N} 2$, and from 2.7 to $4.1 \mathrm{ng} / \mathrm{mL}$ (5.6-8.5 nmol/L) for type B virus, which were collected in a phase II study [12]. Notably, with the lowest single dose $(6 \mathrm{mg})$ tested in the current clinical study, the baloxavir acid $C_{24}(6.92 \mathrm{ng} / \mathrm{mL})$ exceeded the aforementioned target $C_{24}$ in mice. This finding suggests that single oral dosing of baloxavir marboxil could be sufficient to result in a significant, beneficial clinical effect, which is an important consideration from a patient convenience and adherence perspective.

In the food effects study, we found that food state affected the pharmacokinetics of baloxavir acid administered in the form of a single 20-mg tablet. Specifically, in the nonfasted states, exposure was decreased and $\mathrm{CL} / \mathrm{F}$ was increased. Nevertheless, $C_{24}$ still exceeded the target $C_{24}$ in the nonfasted state, indicating that sufficient exposure can be attained in nonfasted states.

\section{Conclusions}

We found that single-dose oral baloxavir marboxil was generally well tolerated, had a favorable safety profile with no particular trend of safety issues among subjects and doses, and had baloxavir acid pharmacokinetic parameters, notably a long half-life, in healthy Japanese male volunteers, supporting single oral dosing. We also found that the AUC of baloxavir acid decreased with food intake; however, the baloxavir acid $C_{24}$ still exceeded the target $C_{24}$. The clinical development of baloxavir marboxil as a novel treatment option for influenza is ongoing.

\section{Compliance with Ethical Standards}

Funding This study was sponsored by Shionogi and Co., Ltd. Medical writing assistance was provided by Luke Carey, $\mathrm{PhD}$, and Rebecca Lew, PhD, CMPP of ProScribe-Envision Pharma Group, and was funded by Shionogi and Co., Ltd. ProScribe's services complied with international guidelines for Good Publication Practice (GPP3).

Conflict of interest Hiroki Koshimichi, Toru Ishibashi, Nao Kawaguchi, Chisako Sato, Akira Kawasaki and Toshihiro Wajima are employees of Shionogi and Co., Ltd.

Ethics approval This study was conducted in accordance with current International Conference on Harmonisation (ICH) Good Clinical Prac- tice (GCP) guidelines, all appropriate subject privacy requirements, and the ethical principles outlined in the Declaration of Helsinki. It was also conducted in accordance with all appropriate regulatory requirements and under a protocol approved by the Institutional Review Board of the study site, Souseikai Hakata Clinic, Fukuoka, Japan.

Informed consent Prior to enrollment, subjects received a full explanation of the nature and objectives of the study, the safety of the drugs under investigation, and that they were free to withdraw from the study at any time without prejudice. Prior to the initiation of studyrelated procedures, all subjects provided written informed consent and received a copy of the signed consent form to keep as a reference.

Open Access This article is distributed under the terms of the Creative Commons Attribution-NonCommercial 4.0 International License (http://creativecommons.org/licenses/by-nc/4.0/), which permits any noncommercial use, distribution, and reproduction in any medium, provided you give appropriate credit to the original author(s) and the source, provide a link to the Creative Commons license, and indicate if changes were made.

\section{References}

1. Paules C, Subbarao K. Influenza. Lancet. 2017;390:697-708.

2. Iuliano AD, Roguski KM, Chang HH, Global Seasonal Influenzaassociated Mortality Collaborator Network, et al. Estimates of global seasonal influenza-associated respiratory mortality: a modelling study. Lancet. 2018;391(10127):1285-300.

3. World Health Organization. WHO fact sheet: influenza (seasonal). 2018. http://www.who.int/mediacentre/factsheets/fs211/en/. Accessed Mar 2018.

4. Ison MG. Antiviral treatments. Clin Chest Med. 2017;38:139-53.

5. Treanor JJ, Hayden FG, Vrooman PS, et al. Efficacy and safety of the oral neuraminidase inhibitor oseltamivir in treating acute influenza: a randomized controlled trial.US Oral Neuraminidase Study Group. JAMA. 2000;283:1016-24.

6. Lee N, Hui DS, Zuo Z, et al. A prospective intervention study on higher-dose oseltamivir treatment in adults hospitalized with influenza A and B infections. Clin Infect Dis. 2013;57:1511-9.

7. Kondo H, Shobugawa Y, Hibino A, et al. Influenza virus shedding in laninamivir-treated children upon returning to school. Tohoku J Exp Med. 2016;238:113-21.

8. Zhang F, Bi Y, Wang J, et al. Human infections with recentlyemerging highly pathogenic H7N9 avian influenza virus in China. J Infect. 2017;75:71-5.

9. Takashita E, Ejima M, Ogawa R, et al. Antiviral susceptibility of influenza viruses isolated from patients pre- and post-administration of favipiravir. Antiviral Res. 2016;132:170-7.

10. Food and Drug Administration Center for Drug Evaluation and Research. Guidance for industry: food-effect bioavailability and fed bioequivalence studies. 2002. https://www.fda.gov/downl oads/drugs/guidancecomplianceregulatoryinformation/guidances/ ucm070241.pdf. Accessed Mar 2018.

11. Noshi T, Satou K, Ishibashi T, et al. Pharmacokinetic and pharmacodynamic analysis of S-033188/S-033447, a novel inhibitor of influenza virus Cap-dependent endonuclease, in mice infected with influenza A virus [abstract]. In: 27th European Congress of Clinical Microbiology and Infectious Diseases (ECCMID); 22-25 April 2017: Vienna.

12. Omoto S, Speranzini V, Hashimoto T, et al. Characterization of influenza virus variants induced by treatment with the endonuclease inhibitor baloxavir marboxil. Sci Rep. 2018;8:article number 9633. 